\title{
Plasmonic Amplification with Ultra-High Optical Gain at Room Temperature
}

SUBJECT AREAS:

NANOPHOTONICS AND PLASMONICS

MICRO-OPTICS

NANOWIRES

PHOTONIC DEVICES

Received

25 March 2013

Accepted

22 May 2013

Published

11 June 2013

Correspondence and requests for materials should be addressed to H.X.X. (hxxu@iphy.ac.

\author{
Ning Liu', Hong Wei', Jing Li', Zhuoxian Wang', Xiaorui Tian' , Anlian Pan² \& Hongxing Xu'
}

${ }^{1}$ Beijing National Laboratory for Condensed Matter Physics and Institute of Physics, Chinese Academy of Sciences, Beijing 100190,
China, ${ }^{2}$ College of Physics and Microelectronics Science, Hunan University, Changsha, 410082, China.

Nanoplasmonic devices are promising for next generation information and communication technologies because of their capability to confine light at subwavelength scale and transport signals with ultrahigh speeds. However, ohmic losses are inherent to all plasmonic devices so that further development of integrated plasmonics requires efficient in situ loss compensation of signals with a wavelength and polarization of choice. Here we show that $\mathrm{CdSe}$ nanobelt $/ \mathrm{Al}_{2} \mathrm{O}_{3} / \mathrm{Ag}$ hybrid plasmonic waveguides allow for efficient broadband loss compensation of propagating hybrid plasmonic signals of different polarizations using an optical pump and probe technique. With an internal gain coefficient of $6755 \mathrm{~cm}^{-1}$ at ambient condition, almost $100 \%$ of the propagation loss of TM-dominant plasmonic signals is compensated. From comparison with a similar photonic structure we attribute the fast-increasing gain at low pump intensity in hybrid plasmonic waveguides to the transfer across the metal-oxide-semiconductor interface of 'hot' electrons photogenerated by the pump light.

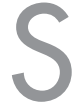

urface plasmon polaritons are coherent oscillations of electrons at metal-dielectric interfaces. They have unique properties that hold promise for potential applications in various fields of science and technology ${ }^{1-3}$. On one hand, plasmonic structures allow overcoming the Abbe diffraction limit and confining light into subwavelength spatial dimensions, thereby opening up for the manipulation of light at the nanoscale. On the other hand, signals carried by surface plasmons can propagate with speeds of the same order as the speed of light. These two properties make nanoplasmonic devices one of the most promising candidates for next-generation information and communication technologies.

However, the integration of plasmon-based components into practical circuits has so far been hindered due to the metallic (ohmic) losses inherent to all plasmonic devices. For example, for surface plasmon propagations whose optical mode is confined in the deep subwavelength region, the signal cannot propagate beyond few tens of micrometers in the absence of loss compensation ${ }^{4}$. Implementing in situ loss-compensation schemes to plasmonic signals can guarantee that information is not lost during transport from one part of a circuit to another and is crucial to the scalability and cascadability of integrated circuits. One possible solution to compensate for losses is through the use of gain materials. Recently, researchers have strived to replace the passive dielectric part of the plasmonic devices with gain media so to compensate for propagation losses ${ }^{5,6}$. Successful in situ loss compensation has been achieved for long-range surface plasmon propagations using fluorescent molecules as the gain medium $^{7,8}$. However, the tradeoff for the obtained loss-compensation is the loss of subwavelength modeconfinement, which is a key requirement for sustaining high integration density.

Here we demonstrate that highly efficient, broadband and polarization-tunable loss compensation of plasmonic signals with optical modes confined in the subwavelength region can be achieved in a semiconductorinsulator-metal hybrid plasmonic waveguide. This type of structure was originally theoretically proposed for its relatively low propagation loss while still maintaining the subwavelength mode confinement ${ }^{9}$ and was recently demonstrated to support lasing from plasmonic modes confined on the nanoscale ${ }^{10}$. However, so far there has been no experimental demonstration of coherent compensation of the propagation loss of an arbitrary input signal carried by the hybrid plasmonic waveguides. Here we experimentally investigate the loss-compensation ability of a hybrid plasmonic waveguide, consisting of a CdSe nanobelt (NB) separated from a silver surface by an $\mathrm{Al}_{2} \mathrm{O}_{3}$ thin layer, to weak probe signals using an optical pump-probe technique and show that these structures support ultra-high optical gain - with gain coefficient exceeding $6755 \mathrm{~cm}^{-1}$ and enough to compensate $99 \%$ of the propagation loss of TM-dominant hybrid plasmonic mode. These values are much higher than the ones observed in ref. 7,8 , but similar to the values calculated in ref. 10. Importantly, we show that the loss-compensation works in the relatively broad spectral band regime and for input signals of orthogonal polarizations, in contrast to nanolasing, which requires single-mode operation ${ }^{10,11}$. 
Our setup also allows us to elucidate the origins of the fast increasing gain at low pump intensity in hybrid plasmonic waveguides. From detailed measurements and comparison of the internal gain in hybrid plasmonic waveguides and their photonic counterparts we found that the transparency threshold, at which the internal gain starts, is 35 times lower in the plasmonic waveguide. Our investigations support our hypothesis that this significant difference and the observed ultra-high optical gain in the hybrid plasmonic waveguides at low pump intensity can be attributed mostly to 'hot' electrons that are generated in the metallic film and are able to cross over the metal-oxide-semiconductor interface to alter the carrier density in the plasmonic waveguide. Our findings help to establish hybrid plasmonic waveguides as a suitable architecture for future integrated plasmonic circuits, and provide valuable insight to their efficient loss-compensation capabilities, potentially enabling their further optimization for plasmon-based information processing.

\section{Results}

Optical gain in a hybrid surface plasmon polariton waveguide. Our hybrid plasmonic waveguide consists of a CdSe NB (with a rectangle cross section) separated from a silver surface by an $\mathrm{Al}_{2} \mathrm{O}_{3}$ thin layer (Fig. 1a and $1 \mathrm{~b}$ ). To allow the detailed investigation of the loss-compensation capabilities of the CdSe NB-based hybrid plasmonic waveguide we use a time-resolved optical pump-probe setup. To that end we used a supercontinuum source $(10 \mathrm{MHz}$ repetition rate, $\sim 72$ picosecond pulse width) to provide both pump and probe light for our experiments (Fig. 1c). The temporal resolution of the measurement was obtained by time-correlated single photon counting (see methods for details).
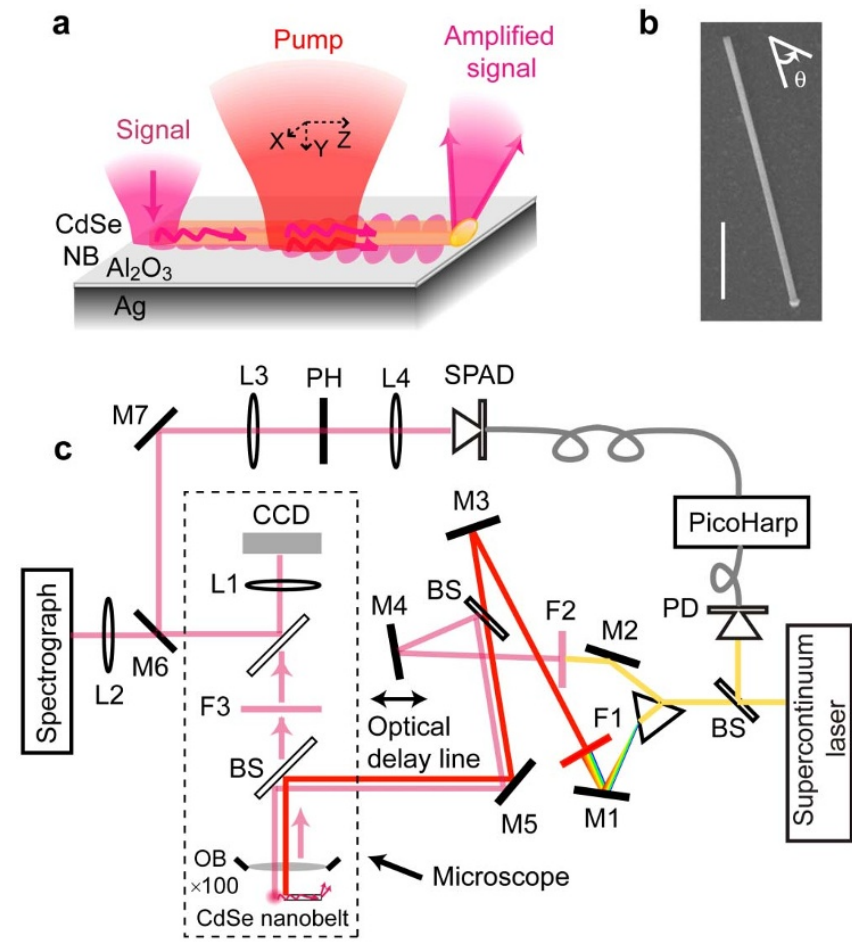

Figure 1 Schematics of the hybrid plasmonic waveguide in compensation mode and experimental setup. (a) Diagram of CdSe $\mathrm{NB} / \mathrm{Al}_{2} \mathrm{O}_{3} / \mathrm{Ag}$ hybrid plasmonic waveguide and the excitation and amplification of input probe signal when operated in pump-probe setup. (b) SEM image of a CdSe NB deposited on the alumina coated Ag film. The scale bar is $2 \mu \mathrm{m}$. (c) Schematics of the experimental setup. BS = beam splitter, $\mathrm{M}=$ mirror, $\mathrm{F}=$ filter, $\mathrm{L}=$ lens, $\mathrm{PH}=$ pin hole, $\mathrm{PD}=$ photo diode, $\mathrm{SPAD}=$ single photon avalanche diode
In our configuration, a probe signal is launched into the waveguide via edge coupling at one end of the NB (see Methods) ${ }^{12}$. This probe signal is loss-compensated by the pump through stimulated emission, and subsequently recorded as scattered photons at the other end of the NB (Fig. 2a, with input laser beam polarized along the long axis of the waveguide) - the dimensions of the $\mathrm{NB}$ are $177 \mathrm{~nm}$ (width) $\times$ $140 \mathrm{~nm}$ (thickness) $\times 8.6 \mu \mathrm{m}$ (length) and $\mathrm{Al}_{2} \mathrm{O}_{3}$ thickness is between 4 to $5 \mathrm{~nm}$. Because the $\mathrm{CdSe} N \mathrm{NB}-\mathrm{Al}_{2} \mathrm{O}_{3}-\mathrm{Ag}$ nanostructure forms a hybrid plasmonic waveguide with tight spatial confinement of the modes in the dielectric gap between CdSe NB and $\mathrm{Ag} \mathrm{film}^{9}$, the input signal is confined to subwavelength dimensions. The pump light was focused onto the centre of the NB (focused beam spot is about $3 \mu \mathrm{m}$ in diameter), spatially separated from the launching spot of the input probe signal. By adjusting the optical delay line in the light path, the waveguided probe signal and pump light can overlap in both space and time yielding an amplification of the input probe signal through stimulated emission. (See sec. 3 of the supplementary information (SI) for details).

The optical images (Fig. 2a) recorded with band pass filter F3 (730 $\pm 5 \mathrm{~nm}$ ), in particular the captures of the output signals (dashed circles in Fig. 2a) clearly show that the output probe signal is significantly amplified when the pump is on (panel III). A corresponding time trace of the intensity of the output (Fig. 2b) further illustrates and quantifies this amplification. To evaluate the internal optical gain of our waveguides, we used the equation ${ }^{13}$

$$
G(\mathrm{~dB})=10 \log \left(\frac{I_{o n}-I_{s p}}{I_{\text {off }}}\right)=10 \log (G)
$$

where $I_{o n}$ is the total output intensity when both probe and pump are on, $I_{s p}$ is the intensity of the spontaneously decayed surface plasmon modes from the pump only, and $I_{\text {off }}$ is the waveguided probe output signal at the end of the NB in the absence of pumping. From the timetrace-intensity measurements results (Fig. 2b) we found that the losscompensated probe signal was 6 times stronger than the uncompensated one, yielding an internal gain of $7.8 \mathrm{~dB}$. One set of spectra (Fig. 2c, taken without the F3 filter) shows that the uncompensated probe signal (red), the spectrum associated with spontaneously decayed surface plasmon modes (blue) and the compensated total signal (green). We can see clearly from the spectra that all wavelengths in the probe signal, spanning over $10 \mathrm{~nm}$, are equally amplified through the stimulated emission, demonstrating the broadband nature of the loss compensation scheme. The slight wiggles on the blue curve are due to resonant cavity modes. Strictly speaking, the gain measured here is not obtained through single path propagation. However, since the wavelengths of the input signal span over $10 \mathrm{~nm}$, covering both peaks and troughs of the cavity modes, the measured average gain over the full wavelength range is very similar to the single path gain at a chosen wavelength. As detailed in the sec. 4 of SI, for the hybrid plasmonic waveguide shown in Fig. 2, the measured average gain is $99 \%$ of the single path gain.

Loss-compensation analysis. Because of its rectangle cross section and dimensions, our NB-based plasmonic device supports multiple hybrid plasmonic modes of different polarizations. Importantly, signals of different polarizations can be loss-compensated efficiently. This is in contrast to the requirements for lasing action, where the polarization of the lasing modes is not easily adjustable once the device has been completed. In our case, the polarization of probe signal can be easily adjusted by changing the polarization of the input laser beam. Internal gain measurements as a function of the pump intensity for probe signals of 90 degree polarization angle difference (Fig. 2d, with input beam polarized along and perpendicular to the waveguide) show clear and significant loss-compensation, exhibiting maximum gain of 8.0 and $8.8 \mathrm{~dB}$, respectively. This corresponds in turn to internal gain coefficients $g$ of $6140 \mathrm{~cm}^{-1}$ and $6755 \mathrm{~cm}^{-1}$, where $g$ is obtained through $G=10 \mathrm{gDlog}(e)$, with $D$ 
a

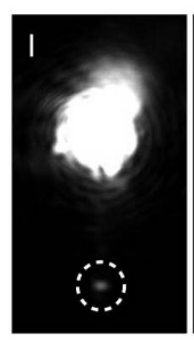

II

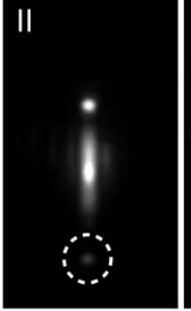

III

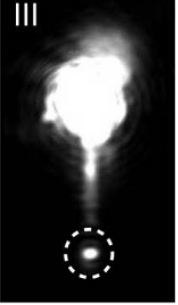

b

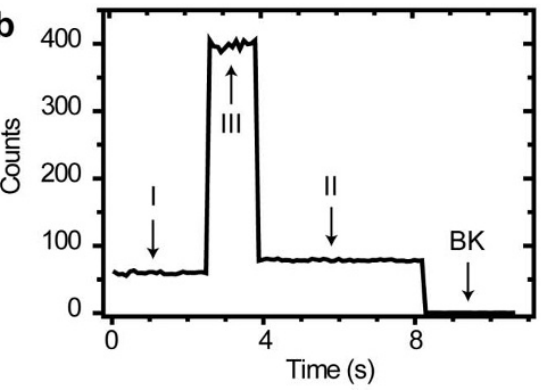

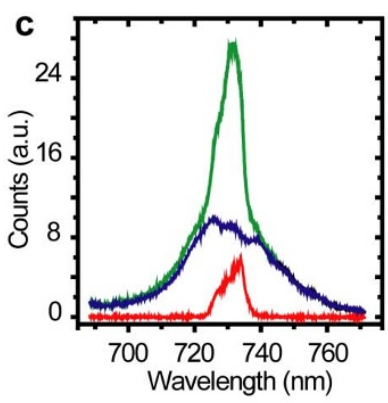
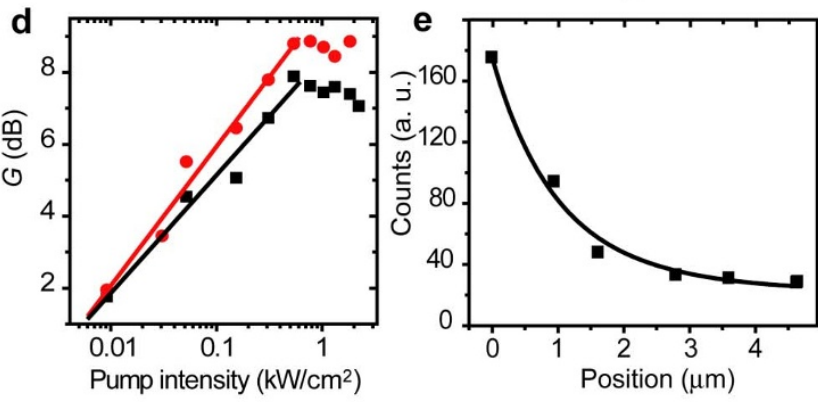

f
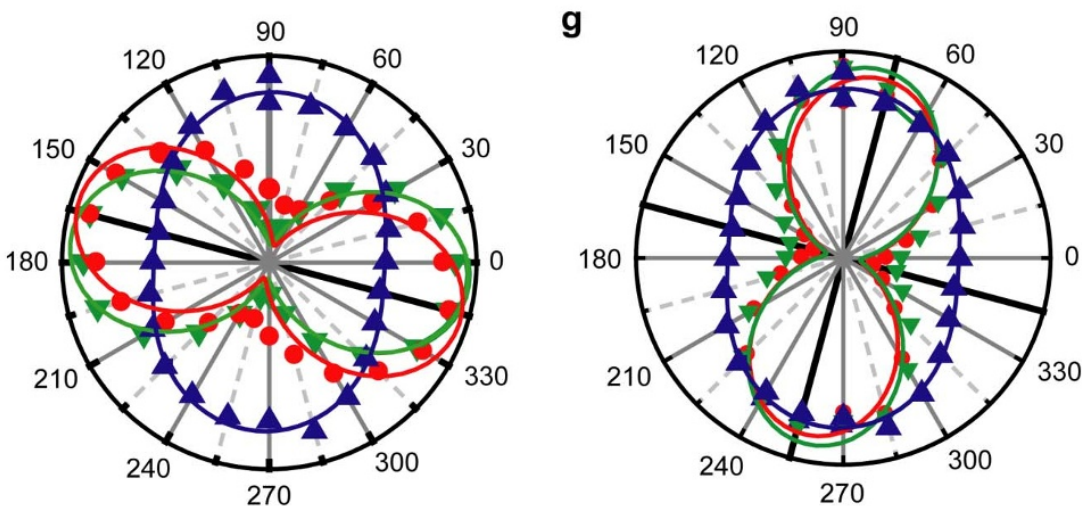

Figure $2 \mid$ Characterization of a hybrid plasmonic waveguide in the compensation mode. (a) Optical images, obtained with $730 \pm 5 \mathrm{~nm}$ band pass filter, corresponding to a probe signal launched from top end of the hybrid plasmonic waveguide (NB $177 \mathrm{~nm} \times 140 \mathrm{~nm} \times 8.6 \mu \mathrm{m})$ and emitted from the bottom end, highlighted by the dashed circle (I), photoluminescence (PL) with the pump only (II), and the amplification of the probe signal when both pump and probe are present (III). The two ends of the waveguide are clearly visible as the top and bottom bright spots in (II). (b) Time trace of the output intensity at the emission end of the hybrid plasmonic waveguide. The dashed circles in (a) mark the area where the time trace was obtained. BK indicates the background dark counts. (c) Typical set of emission spectra, representing the probe signal (red), PL (blue), and total output (green). The pump intensity is $0.77 \mathrm{~kW} / \mathrm{cm}^{2}$. (d) Gain $G(\mathrm{~dB})$.vs. pump intensity. ' $\mathbf{\square}$ ' and ' $\bullet$ ' correspond to the measured gains of input laser beam polarized along and perpendicular to the waveguide respectively and the black and red lines corresponds to logarithmic fits to the $G$ value at pump intensity lower than $0.6 \mathrm{~kW} / \mathrm{cm}^{2}$. (e) Propagation loss measurement. Output light intensity as a function of pump position and fit to linear combination of two exponential decay functions. (f-g) Plots of the emission polarization with input laser beam polarized in $0^{\circ}$ and $90^{\circ}$ respectively and pump polarized at $0^{\circ}$. Red, blue and green symbols describe the polarization of the emitted probe signal, scattered photons from spontaneous decayed SPs excited by the pump, and total amplified signal, respectively. Polar angle corresponds to angle $\theta$ defined in Fig. $1 \mathrm{~b}$. The curves are normalized to the maximum intensity. Red and green curves are fits to linear polarized lights. Blue curve is the fit to linear combination of two non-coherent orthogonally linearly polarized lights.

the diameter of pump beam. At the pump intensity lower than $500 \mathrm{~W} / \mathrm{cm}^{2}$, we found that the gain $G(\mathrm{~dB})$ increases monotonically and in a highly non-linear fashion with increasing pump intensity. To confirm the nonlinear dependence we investigated the intensity of spontaneously decayed surface plasmon modes as a function of the pump intensity (See sec. 5 of the SI), which does not exhibit a linear dependence on the pump intensity either.

The nonlinearity in both gain and spontaneous emission measurement both suggest that the single-exciton recombination mechanism cannot give a satisfactory explanation to our experimental data. Instead, the measured gain $G(\mathrm{~dB})$ follows logarithmic dependence on the pump intensity, as indicated by the fits in Fig. $2 \mathrm{~d}$, similar to that of semiconductor quantum well structures ${ }^{14,15}$. Fitting the gain data to the empirical equation $G=G_{0} \ln \frac{I}{I_{t r}}$, where $I_{t r}$ denotes the transparency pump intensity, yields $I_{t r}=2.8 \mathrm{~W} / \mathrm{cm}^{2}$ and $G_{0}=$ 1.42 and 1.68 for input laser beam polarized along and perpendicular to the waveguide, respectively. Note here that the $I_{t r}$ is not lasing threshold, but a value indicating the start of gain. This fast gain increase at low pump intensity is further investigated in the later sections. Since $G(\mathrm{~dB})$ is proportional to the internal modal gain coefficient $g$, this means that $g$ is slightly higher for the perpendicularly polarized mode. In a simple model, the modal gain coefficient $g$ can be determined by the product of $f g_{m}$, where $f$ is the fractional overlap of the electric field excitation profile of the CdSe NB with the spatial dimension of waveguided mode of the probe signal, and $g_{m}$ is the material gain coefficient. Because $g_{m}$ is the same for the same structure, the difference in the modal gain is determined by the fractional overlap factor $f$ of these two polarizations. 

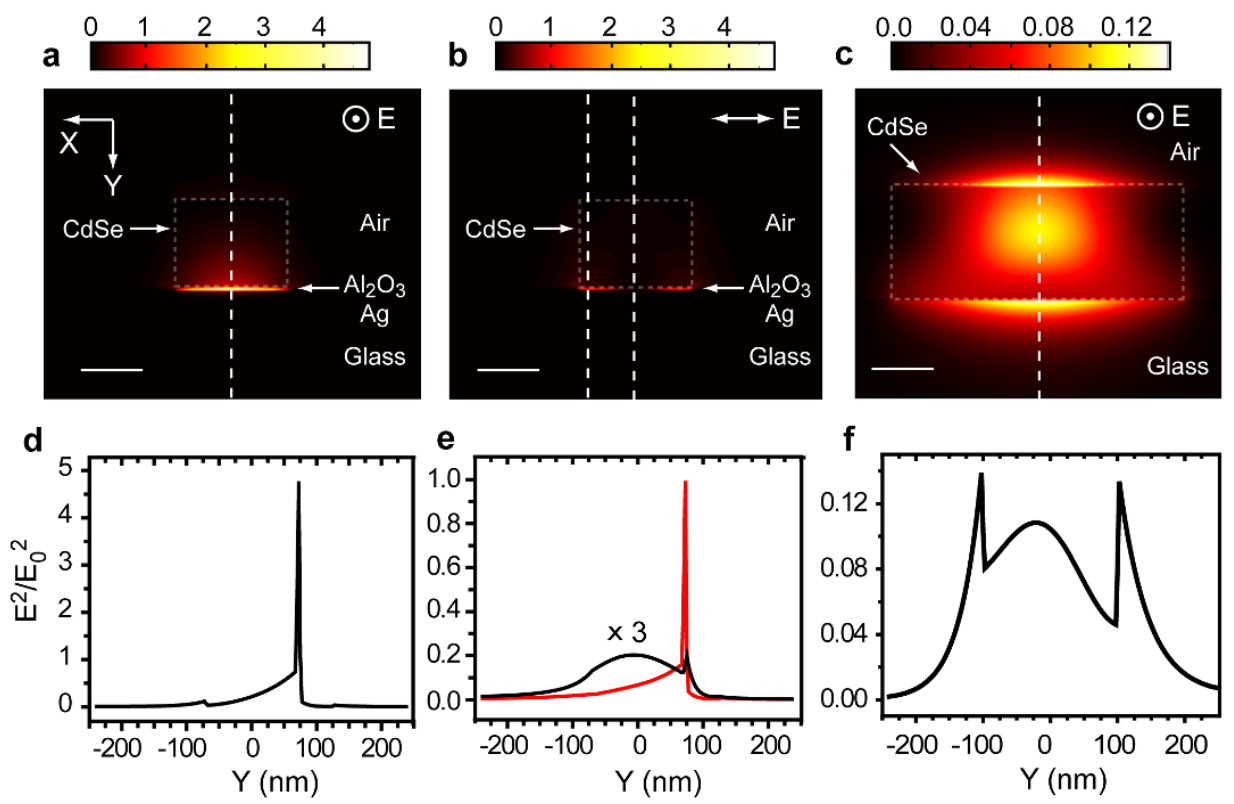

Figure 3 FDTD simulation of electric field intensity distribution within the plasmonic and photonic structures. (a-b) The hybrid plasmonic modes excited in a hybrid plasmonic waveguide with incident beam polarized along Z direction (a) and X direction (b). The dimension of the CdSe NB cross section is $177 \mathrm{~nm} \times 140 \mathrm{~nm}$. (c) The photonic mode excited in a photonic NB, with incident beam polarized along Z direction. The dimension of the photonic NB cross section is $468 \mathrm{~nm} \times 198 \mathrm{~nm}$. The scale bars in $(\mathrm{a}-\mathrm{c})$ indicate $100 \mathrm{~nm}$. (d-f) The corresponding plots of the field intensity .vs. Y position, following the white dashed lines highlighted in (a) to (c). The black curve in (e) is obtained along the dashed line in the center and the red curve along the dashed line off center. The XY planes correspond to $Z=2 \mu \mathrm{m}$.

To obtain $f$ for plasmonic mode of both polarizations, we have simulated the electric field intensity distribution of the waveguided modes over the cross section of the NB (perpendicular to the long axis of the waveguide) ${ }^{16}$. Same as our experiments, the waveguided plasmonic modes in our simulation were also excited by edge coupling with input laser beam polarized along and perpendicular to the waveguide. In the simulation, we choose the origin at the centre of the waveguide, $\mathrm{Z}$ along the long axis of the waveguide, and $\mathrm{Y}$ perpendicular to the semiconductor-insulator-metal interface. From our calculated results (Figs. 3a, b) it is clear that the electric field is strongly concentrated in the $\mathrm{Al}_{2} \mathrm{O}_{3}$ layer for the hybrid plasmonic modes. To better illustrate the variation of the electric field in the $\mathrm{Y}$ direction, the intensities as a function of $\mathrm{Y}$ position along the dashed lines in Fig. 3a and 3b, are shown in Fig. 3d and 3e. We also note that the electric field intensity distributions in Fig. $3 \mathrm{a}$ and $3 \mathrm{~b}$ show strong TM and TE-dominant characters for input beam polarized along and perpendicular to the waveguide, respectively, similar to the $\mathrm{m}=0$ and $\mathrm{m}=1$ plasmonic modes supported by Ag nanowires ${ }^{17}$. By calculating the ratio of the integral of the field intensity over the area within the NB with that over the entire spatial expansion of the waveguided mode, we obtain the fractional factor $f$ for the TM and TE-dominant modes are 0.64 and 0.66 respectively. The $f$ for the mode (TE-dominant) excited by the perpendicular polarized input beam is slightly higher than that for the other polarization direction (TM-dominant), so is its modal gain.

The gain plateaus at pump intensities above $500 \mathrm{~W} / \mathrm{cm}^{2}$. Because the gain $G(\mathrm{~dB}$ ) only decreases at most $16 \%$ (in $\mathrm{dB}$ ) with a 50 -fold increase of the probe signal intensity (See sec. 6 of the SI) we conclude that the saturation most likely can be attributed to higher-order nonradiative processes, such as Auger recombination, which dominates at high pump intensity.

The polarizations of the waveguided probe signals excited by input beam polarized along and perpendicular to the waveguides were measured experimentally at the output end of the waveguide, which show linear polarization along -10 degree and 80 degrees (Fig. $2 \mathrm{f}$ and $2 \mathrm{~g}$ ), respectively. The slight polarization rotation with respect to the excitation laser polarization we obtained on the probe signals is most likely caused by the attachment of the Au catalyst at the emission end of the waveguide. The compensated probe signals were also measured. As expected, the polarization of the compensated signal follows the polarization of the uncompensated probe within experimental errors. To estimate the propagation loss in the hybrid plasmonic structure, we used medium $\left(\sim 250 \mathrm{~W} / \mathrm{cm}^{2}\right)$ pump intensity to excite the hybrid plasmonic waveguide from the middle of the waveguide with a beam diameter of $3 \mu \mathrm{m}$. By moving the pump spot along the NB and recording the intensity of the photons at the output end of NB, stemming from the spontaneously decayed surface plasmon modes, we can obtain a plot of the output intensity. vs. pump location (Fig. 2e). The propagation loss coefficients can be extracted from the decay curve. Both TM ( -10 degree) and TE (80 degree) dominant modes contribute to the loss measurement. To quantify the contribution from each mode, we measured the polarization of the spontaneously decayed output light (blue), as shown in Fig. 2f. Assuming the total intensity of the spontaneously decayed light follows $I_{\text {total }}=I_{-10} \cos ^{2}(\theta+10)+I_{80} \cos ^{2}(\theta-80)$, where $I_{-10}$ and $I_{80}$ denote the contribution from TM and TE-dominant modes, we obtain the ratio of $I_{-10}: I_{80}=1: 1.38$. Fitting the decay data into the linear combination of two exponential decay functions with appropriate weighting factor (Fig. 2e), we find that the measured propagation loss coefficients for the TM and TE dominant modes are $6230 \mathrm{~cm}^{-1}$ and $11420 \mathrm{~cm}^{-1}$, respectively. Compared to the measured internal gain for each mode, the propagation loss was compensated $99 \%$ for the TM dominant mode and 59\% for the TE dominant mode at ambient condition. Since the total signal loss in the waveguides includes both propagation loss and transmission loss, even though the propagation loss was almost fully compensated for the TM dominant mode in our experiment, the transmission loss at both ends of the waveguides (could be $30 \sim 70 \%$, see sec. 4 and 10 of SI) stopped our hybrid plasmonic waveguides from lasing at room temperature. We note that the calculated propagation loss coefficients for TM and TE modes are $5624 \mathrm{~cm}^{-1}$ and $10309 \mathrm{~cm}^{-1}$, respectively (See sec. 8 of SI), 11\% lower than the measured ones, probably due to 


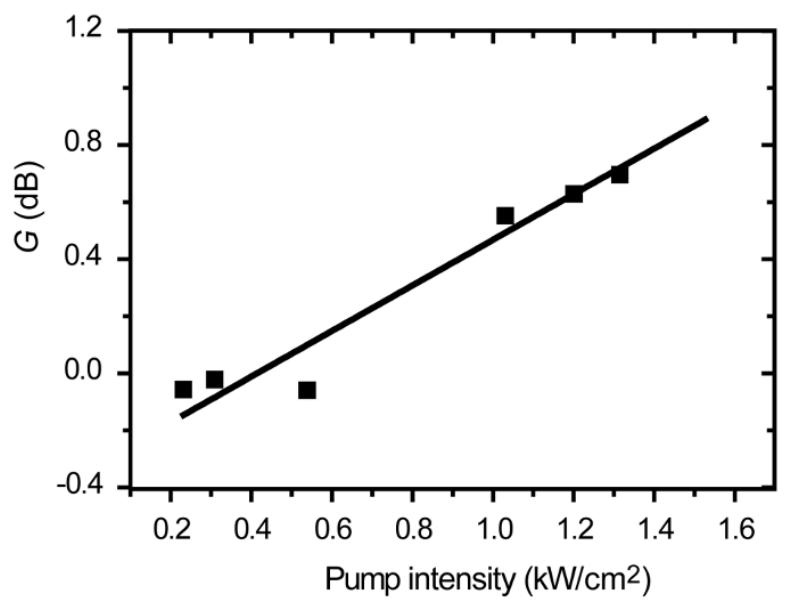

Figure $4 \mid$ Characterization of the gain of a photonic amplifier. $\mathrm{G}(\mathrm{dB})$.vs. Pump intensity plot of a photonic waveguide. Black line is the linear fit of $\mathrm{G}(\mathrm{dB})$ to pump intensity.

the poor quality of the thermal evaporated Ag film. Not surprisingly, the propagation loss coefficient for the TE-dominant hybrid plasmonic mode is much larger than that of the TM mode. This is why the lasing mode reported previously in the hybrid plasmonic waveguide is TM-dominant ${ }^{8}$. Operated in the pump-probe compensation configuration, however, the same plasmonic structure can be used to compensate signals of either mode.

Comparison with gain of CdSe NB photonic waveguide. To understand the origin of the fast optical gain increase observed in our hybrid plasmonic waveguides at low pump intensity, we used the same pump-probe technique to directly evaluate and compare the gain coefficient of the active material in a photonic waveguide configuration. To that end we fabricated photonic waveguides from CdSe NBs through direct drop-casting onto glass sides. For CdSe NBs thinner than $160 \mathrm{~nm}$, no loss compensation can be obtained due to the weak mode overlap with the gain material. For thicker CdSe NBs, most of them start to lase at medium pump intensity. The lasing from photonic waveguides sometimes even causes the measured gain to undergo an abrupt transition. (See sec. 9 to 11 of SI) Therefore, we only measured the optical gain of these photonic waveguides up to their lasing threshold, which is usually below $800 \mathrm{~W} / \mathrm{cm}^{2}$ and the gain coefficients are found much lower than that for hybrid plasmonic waveguides at the same pump intensity.

Fig. 4 shows a plot of measured gain $G(\mathrm{~dB})$ as a function of pump intensity on one photonic waveguide $(468 \mathrm{~nm} \times 198 \mathrm{~nm} \times$ $14.5 \mu \mathrm{m})$. Comparing the gain of the plasmonic and photonic waveguides described in Figs. 2 and 4, it is clear that the gain coefficient, $g$, for the hybrid plasmonic waveguide is substantially higher than that of the photonic case for the same pump intensity. Moreover, the transparency pump intensity, at which $G(\mathrm{~dB})=0$, is at least $300 \mathrm{~W} / \mathrm{cm}^{2}$ for the photonic structure in Fig. 4, which is more than 107 times the value of $2.8 \mathrm{~W} / \mathrm{cm}^{2}$ obtained for the plasmonic case.

To understand the 107-fold reduction in the transparency pump intensity in the hybrid plasmonic waveguide, we have investigated the field intensity distribution of the waveguided photonic mode with input laser beam polarized along photonic waveguide (Fig. 3c). Using the same method as before, we obtain a fractional mode factor $f=0.52$, which is only $21 \%$ smaller than the plasmonic counterpart. This implies that the large difference in modal gain (determined by $f g_{m}(I)$ ) must originate from the difference in the material gain at the same pump intensity for these two configurations. Because the material gain for semiconductors is determined by the carrier density at a specific pump intensity and the stimulated emission cross section, assuming the same stimulated emission cross section in these two cases, we conclude that the modal gain difference observed in the plasmonic and photonic waveguides can be attributed to different carrier densities being generated at the same pump intensity.

Although the absolute value of the carrier density $N$ for each NB is difficult to measure, we can estimate the electric field intensity within the gain medium during pumping. Figure $5 \mathrm{a}$ and $5 \mathrm{~b}$ show the simulation results of the intensity distribution on the $\mathrm{YZ}$ plane at $\mathrm{X}=0$ for the hybrid plasmonic and photonic case respectively, with the pump polarized in the $\mathrm{Z}$ direction (the length of the NB is set to $5 \mu \mathrm{m}$ ). The averaged intensity within the NB over the pumped region is $1.03 I_{0}$ and $0.44 I_{0}$ for the hybrid plasmonic and photonic case respectively, where $I_{0}$ is the initial pump intensity. Assuming the same absorption cross section of CdSe in these two configurations, the ratio of the pump rate $R_{p l} / R_{p h}$ is equal to the ratio of the field intensity, which is 2.34 in this case. According to our analyses (See sec. 5 in the SI), the carrier density has a linear dependence on the pump rate at low pump intensities. Therefore, the ratio of carrier density directly generated within the gain materials, $N_{p l} / N_{p h}$, is also equal to 2.34 . However, this value and the fractional factor $f$ together still cannot account for the 107-fold reduction of the transparency pump intensity in the hybrid plasmonic waveguide in comparison to its photonic counterpart. Neither can potential variations in the quality of the NBs. While we have seen slight variations between samples the averaged transparency threshold for the photonic waveguides is $150 \mathrm{~W} /$ $\mathrm{cm}^{2}$, which is 36 times higher than that for the hybrid plasmonic cases, which is around $4.1 \mathrm{~W} / \mathrm{cm}^{2}$. Other mechanisms must therefore be involved in explaining the relatively high optical gain observed in the hybrid plasmonic waveguides at low pump energy.

\section{Discussion}

We argue that the origin of the observed ultra-high optical gain in the hybrid plasmonic waveguide can be attributed to photogenerated 'hot' electrons that transfer from the Ag film to the NB, thereby altering its carrier density. In a recent paper published by $\mathrm{Ma}$ et al. ${ }^{18}$, the authors electrically modulated the electron-hole pairs in the gain material $\mathrm{CdS}$ (in the $\mathrm{CdS} / \mathrm{MgF}_{2} / \mathrm{Ag}$ geometry) and they observed that the Spaser output intensity changed dramatically with the variation of carrier density in the gain material, demonstrating that by changing the carrier density in the gain materials, the condition of stimulated emission can be altered substantially.

It is well known that when a metal is in direct contact with a semiconductor, Schottky barrier is formed at the interface due to the difference in work function between the two materials ${ }^{19,20}$. Photodetectors based on the photogenerated carriers transporting through the Schottky barrier have been realized in Ag-CdSe and Graphene-CdSe NB structures ${ }^{21,22}$. In both cases, photogenerated 'hot' electrons were observed to flow into CdSe from the other side of the Schottky barrier at zero bias. For the plasmonic structure we studied here, a 4 to $5 \mathrm{~nm}$ thick $\mathrm{Al}_{2} \mathrm{O}_{3}$ layer is formed between the Ag$\mathrm{CdSe}$ interface. Depending on the growth temperature, the metal$\mathrm{Al}_{2} \mathrm{O}_{3}$ barrier height can vary from 1.2 to $3.2 \mathrm{eV}$ for $\mathrm{Al}_{2} \mathrm{O}_{3}$ layer with a thickness ranging from $1.5 \mathrm{~nm}$ to $8.5 \mathrm{~nm}^{23-25}$. A barrier height of $1.72 \mathrm{eV}$ have been reported at $\mathrm{Ag}-\mathrm{Al}_{2} \mathrm{O}_{3}$ interface for $\mathrm{Al}_{2} \mathrm{O}_{3}$ layer formed at $70^{\circ} \mathrm{C}^{26}$. In our studies, the $\mathrm{Al}_{2} \mathrm{O}_{3}$ was grown at $200{ }^{\circ} \mathrm{C}$ and the energy of the pump photons is between 1.85 to $1.97 \mathrm{eV}$. It is very possible that the photogenerated 'hot' electrons in Ag film have gained enough energy to transit through the conduction band of the $\mathrm{Al}_{2} \mathrm{O}_{3}$ layer and reach CdSe NB. The extra electrons will accumulate at the $\mathrm{Al}_{2} \mathrm{O}_{3}-\mathrm{CdSe}$ interface to build up a potential barrier in order to slow down the further transport of 'hot' electrons from the Ag side. The electron flow will continue until a new equilibrium is established. The accumulated charge carriers at the $\mathrm{Al}_{2} \mathrm{O}_{3}$-CdSe interface substantially alter the electron-hole distribution as well as the carrier density in the CdSe NB. Because the largest hole 


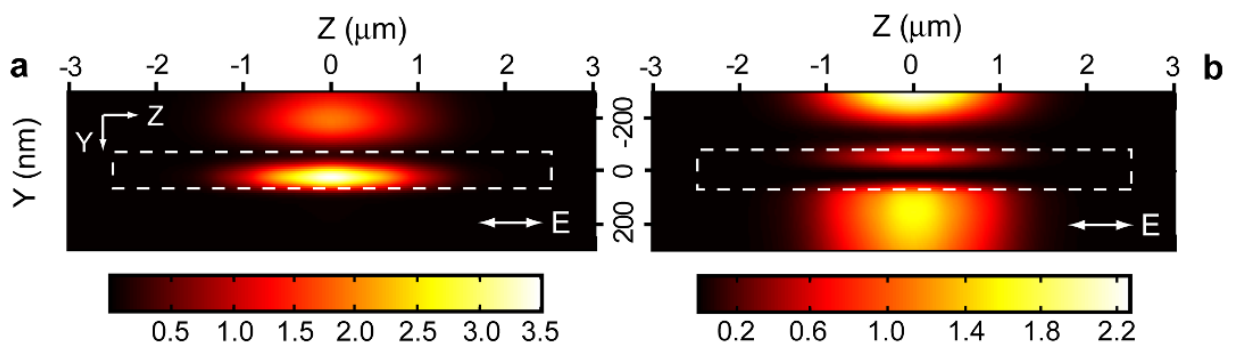

Figure 5 FDTD simulation of electric field intensity distribution within the plasmonic and photonic waveguides when being pumped at the middle. (a-b) The simulations of electric field intensity distribution on $\mathrm{YZ}$ plane at $\mathrm{X}=0 \mu \mathrm{m}$, when the pump light at wavelength $650 \mathrm{~nm}$ is incident on the middle of the NB, for plasmonic (Fig. 3a) and photonic waveguide (Fig. 3c), respectively. The incident beam is polarized in $\mathrm{Z}$ direction.

population also exists at the $\mathrm{Al}_{2} \mathrm{O}_{3}$-CdSe interface due to the Schottky barrier, the charge transfer can greatly reduce the threshold pump intensity needed to reach the population inversion within the plasmonic waveguides (in other words, effectively increase the pump rate), especially at the interface.

To verify that the charge transfer is viable through the ultrathin $\mathrm{Al}_{2} \mathrm{O}_{3}$ film, we measured the current as a function of applied voltage across a $\mathrm{Ag}-\mathrm{Al}_{2} \mathrm{O}_{3}-\mathrm{Ag}$ junction. The measurements yield surprisingly low resistance across the $\mathrm{Ag}-\mathrm{Al}_{2} \mathrm{O}_{3}-\mathrm{Ag}$ junction, $4.89 \mathrm{ohm}$ for a $4 \mathrm{~nm}$ thick $\mathrm{Al}_{2} \mathrm{O}_{3}$ film with the top electrode around $300 \mu \mathrm{m}$ in diameter, for bias voltage from 1 to $50 \mathrm{mV}$ (in both polarities). (See sec. 12 of SI for details) These results suggest that the 'hot' electrons can easily transfer from the metal to the CdSe nanobelt up on light radiation.

The hot-electron transfer across the $\mathrm{Al}_{2} \mathrm{O}_{3}$-CdSe interface can also explain the fast gain increase at lower pump intensity for the plasmonic waveguide. Because the amount of extra charge needed to reestablish the equilibrium at the interface is fixed, their contribution to the carrier density is more significant at lower pump intensity. This explanation is further supported by the linear behaviour of the gain $G(\mathrm{~dB})$.vs. the pump intensity in the photonic case (Fig. 4), where the charge transfer is absent. Increasing the thickness of $\mathrm{Al}_{2} \mathrm{O}_{3}$ film can lead to the up-shift of the conduction band of the $\mathrm{Al}_{2} \mathrm{O}_{3}$ and in turn increase the transparency threshold of the plasmonic waveguides. Our experimental results did show an increasing trend in the transparency threshold as the $\mathrm{Al}_{2} \mathrm{O}_{3}$ film thickness of the hybrid plasmonic waveguides changed from 5 to $15 \mathrm{~nm}$, consistent with our expectation (See sec. 13 of SI).

In conclusion, the implementation of pump to compensate for the propagation loss of any nanoscale hybrid plasmonic probe signals can greatly extend the propagation length of the signal within a plasmonic device and offer solutions to one of the largest obstacles that hinder the further development of integrated plasmon-based circuitry. The fast increase in internal gain at low pumping intensity also sheds light on the potential application of high gain, low threshold plasmonic amplifier and lasers in the future.

\section{Methods}

Sample preparation. CdSe NBs $(40 \sim 300 \mathrm{~nm}$ thick, $100 \sim 500 \mathrm{~nm}$ wide, and $5 \sim 20 \mu \mathrm{m}$ long) were formed on the Si substrate with chemical vapor deposition (CVD). After growth, the CdSe NBs were suspended in ethanol solution for use. Smooth Ag films with the thickness of 40 to $60 \mathrm{~nm}$ were thermally evaporated onto the glass cover slides (Roughness of the film is about $1 \mathrm{~nm}$, see supplementary information for details). An ultrathin and ultrasmooth $\mathrm{Al}_{2} \mathrm{O}_{3}$ layer of 4 to $5 \mathrm{~nm}$ was then grown on top of the Ag film by atomic layer deposition (ALD) method at $200^{\circ} \mathrm{C}$. Finally, the CdSe NB suspensions were drop-casted onto the alumina coated Ag film. For some samples, another protection layer of $\mathrm{Al}_{2} \mathrm{O}_{3}$ of $3 \mathrm{~nm}$ was grown on top of the structure.

Optical measurements. A supercontinuum light source $(10 \mathrm{MHz}$ repetition rate, $\sim 72$ picosecond pulse width, Fianium) is used to provide both pump and probe for our experiments. Part of the output light from the supercontinuum was used to provide the reference input to the counting unit Picoharp 300 (PicoQuant). The rest of the output was further split into two beams. The one with majority of the power was utilized as pump light. A band pass filter was used to limit the wavelength of the pump light to $650 \pm 20 \mathrm{~nm}$. The beam with small portion of the power was employed as the probe. Because the emission peak of our CdSe NBs is mostly centred on $730 \mathrm{~nm}$, we applied another band pass filter to restrict the wavelength of the probe light to be $730 \pm 5 \mathrm{~nm}$ in order to spectrally overlap with the emission band of the gain medium. The two beams were then directed into an upright microscope (Olympus), and finally focused onto the sample by a $\times 100$ objective $(\mathrm{NA}=0.9)$. The probe signal is coupled into our waveguide through edge coupling. Different from the end-fired configuration, in which case the input laser beam would travel in the same direction as the waveguide, in our case the input laser beam is travelling perpendicular to the long axis of the waveguide and is focused at one end of the waveguide with a focused beam diameter around $3 \mu \mathrm{m}$. The field of view of the sample with the $\times 100$ objective is more than $100 \mu \mathrm{m} \times 100 \mu \mathrm{m}$. Light emitted from this area can be collected by the same objective. Our waveguides are only few tens of microns long. Light emitted from these waveguides can be easily re-collected by the same objective, and be selectively directed to a CCD camera (DVC), a spectrograph (Acton, Princeton Instruments), or a single photon avalanche diode detector (SPAD).

1. Gramotnev, D. K. \& Bozhevolnyi, S. I. Plasmonics beyond the diffraction limit. Nature Photon. 4, 83-91 (2010).

2. Atwater, H. A. \& Polman, A. Plasmonics for improved photovoltaic devices. Nature Mater. 9, 205-213 (2010).

3. Schuller, J. A. et al. Plasmonics for extreme light concentration and manipulation. Nature Mater. 9, 193-204 (2010).

4. Ditlbacher, H. et al. Silver Nanowires as Surface Plasmon Resonators. Physical Review Letters 95, 257403 (2005).

5. Berini, P. \& De Leon, I. Surface plasmon-polariton amplifiers and lasers. Nature Photon. 6, 16-24 (2012).

6. Lu, Y. J. et al. Plasmonic Nanolaser Using Epitaxially Grown Silver Film. Science 337, 450-453 (2012).

7. Gather, M. C., Meerholz, K., Danz, N. \& Leosson, K. Net optical gain in a plasmonic waveguide embedded in a fluorescent polymer. Nature Photon. 4, 457-461 (2010).

8. De Leon, I. \& Berini, P. Amplification of long-range surface plasmons by a dipolar gain medium. Nature Photon. 4, 382-387 (2010).

9. Oulton, R. F., Sorger, V. J., Genov, D. A., Pile, D. F. P. \& Zhang, X. A hybrid plasmonic waveguide for subwavelength confinement and long-range propagation. Nature Photon. 2, 496-500 (2008).

10. Oulton, R. F. et al. Plasmon lasers at deep subwavelength scale. Nature 461, 629-632 (2009)

11. Ma, R. M., Oulton, R. F., Sorger, V. J., Bartal, G. \& Zhang, X. A. Room-temperature sub-diffraction-limited plasmon laser by total internal reflection. Nature Mater. 10, 110-113 (2011).

12. Li, Z. P. et al. Directional Light Emission from Propagating Surface Plasmons of Silver Nanowires. Nano Lett. 9, 4383-4386 (2009).

13. Liu, N., Ruseckas, A., Montgomery, N. A., Samuel, I. D. W. \& Turnbull, G. A. Semiconducting polymer waveguides for end-fired ultra-fast optical amplifiers. Opt. Express 17, 21452-21458 (2009).

14. Svelto, O. Principles of lasers. $4^{\text {th }}$ edn, (Springer, 2009).

15. Suhara, T. Semiconductor laser fundamentals. (Marcel Dekker, Inc., 2004).

16. Visser, T. D., Blok, H., Demeulenaere, B. \& Lenstra, D. Confinement factors and gain in optical amplifiers. IEEE Journal of Quantum Electronics 33, 1763-1766 (1997).

17. Li, Z. P. et al. Correlation between incident and emission polarization in nanowire surface plasmon waveguides. Nano Lett. 10, 1831-1835 (2010).

18. Ma, R. M., Yin, X. B., Oulton, R. F., Sorger, V. J. \& Zhang, X. Multiplexed and Electrically Modulated Plasmon Laser Circuit. Nano Lett. 12, 5396-5402 (2012).

19. Sze, S. M. \& Ng, K. K. Physics of semiconductor devices. $3^{\text {rd }}$ edn, (John Wiley \& Sons, Inc., 2007).

20. Knight, M. W., Sobhani, H., Nordlander, P. \& Halas, N. J. Photodetection with Active Optical Antennas. Science 332, 702-704 (2011).

21. Sharma, S. K., Kumar, S., Kumar, V. \& Sharma, T. P. CdSe photovoltaic sintered films. Opt. Mater. 13, 261-265 (1999) 
22. Jin, W. F. et al. Self-powered high performance photodetectors based on CdSe nanobelt/graphene Schottky junctions. J. Mater. Chem. 22, 2863-2867 (2012).

23. Rippard, W. H., Perrella, A. C., Albert, F. J. \& Buhrman, R. A. Ultrathin aluminum oxide tunnel barriers. Phys. Rev. Lett. 88, 046805 (2002).

24. Kaltenbrunner, M. et al. Anodized Aluminum Oxide Thin Films for RoomTemperature-Processed, Flexible, Low-Voltage Organic Non-Volatile Memory Elements with Excellent Charge Retention. Adv. Mater. 23, 4892-4896 (2011).

25. Afanas'ev, V. V., Houssa, M., Stesmans, A. \& Heyns, M. M. Band alignments in metal-oxide-silicon structures with atomic-layer deposited $\mathrm{Al}_{2} \mathrm{O}_{3}$ and $\mathrm{ZrO}_{2}$. J. Appl. Phys. 91, 3079-3084 (2002).

26. Singh, K. \& Hammond, S. N. A. Current-Voltage Characteristics and Photoresponse of Metal $\mathrm{Al}_{2} \mathrm{O}_{3}$ Metal Devices. Tr. J. of Phys. 22, 315-323 (1998).

\section{Acknowledgments}

This work was supported by The Ministry of Science and Technology of China Grants 2009CB930700 and 2012YQ12006005, National Natural Science Foundation of China

Grants 11134013, 11004237 and 11227407, and "Knowledge Innovation Project"

(KJCX2-EW-W04) of Chinese Academy of Sciences (CAS). The authors thank the

Laboratory of Microfabrication in the Institute of Physics of CAS for experimental support.

\section{Author contributions}

N. L. and H. X. X. designed the experiments. A. L. Pan provided the CdSe nanobelts. N. L., J. L., Z.X. W. and X. R. T. fabricated the samples. N. L. performed the experiments, analyzed the data, and did the numerical simulations. N. L., H. W. and H. X. Xu wrote the manuscript together and all authors reviewed the manuscript.

\section{Additional information}

Supplementary information accompanies this paper at http://www.nature.com/ scientificreports

Competing financial interests: The authors declare no competing financial interests.

How to cite this article: Liu, N. et al. Plasmonic Amplification with Ultra-High Optical Gain at Room Temperature. Sci. Rep. 3, 1967; DOI:10.1038/srep01967 (2013).

(c) (i) $\Theta$ This work is licensed under a Creative Commons Attribution-

cc. license, visit http://creativecommons.org/licenses/by-nc-nd/3.0 